\title{
BROOKHTUEN
}

NATIONAL LABORATORY

BNL-96751-2012-CP

\section{Simultaneous global coupling and vertical dispersion correction in RHIC}

\author{
C. Liu, Y. Luo, M. Minty
}

Presented at the International Particle Accelerator Conference 2012 (IPAC'12)

New Orleans, LA

May 20-25, 2012

\section{Collider-Accelerator Department \\ Brookhaven National Laboratory \\ U.S. Department of Energy DOE Office of Science}

\footnotetext{
Notice: This manuscript has been authored by employees of Brookhaven Science Associates, LLC under Contract No. DE-AC02-98CH10886 with the U.S. Department of Energy. The publisher by accepting the manuscript for publication acknowledges that the United States Government retains a non-exclusive, paid-up, irrevocable, world-wide license to publish or reproduce the published form of this manuscript, or allow others to do so, for United States Government purposes.

This preprint is intended for publication in a journal or proceedings. Since changes may be made before publication, it may not be cited or reproduced without the author's permission.
} 


\section{DISCLAIMER}

This report was prepared as an account of work sponsored by an agency of the United States Government. Neither the United States Government nor any agency thereof, nor any of their employees, nor any of their contractors, subcontractors, or their employees, makes any warranty, express or implied, or assumes any legal liability or responsibility for the accuracy, completeness, or any third party's use or the results of such use of any information, apparatus, product, or process disclosed, or represents that its use would not infringe privately owned rights. Reference herein to any specific commercial product, process, or service by trade name, trademark, manufacturer, or otherwise, does not necessarily constitute or imply its endorsement, recommendation, or favoring by the United States Government or any agency thereof or its contractors or subcontractors. The views and opinions of authors expressed herein do not necessarily state or reflect those of the United States Government or any agency thereof. 


\title{
Simultaneous global coupling and vertical dispersion correction in RHIC*
}

\author{
C. Liu ${ }^{\dagger}$, Y. Luo, M. Minty, BNL, Upton, NY 11973, USA
}

\section{Abstract}

Residual vertical dispersion on the order of $+/-0.2 \mathrm{~m}$ (peak to peak) has been measured at store energies for both polarized protons and heavy ion beams in RHIC. The hypothesis is that this may have impact on the polarization transmission efficiency during the energy ramp, the polarization lifetime at store and, for heavy ions, the dynamic aperture. An algorithm to correct global coupling and dispersion simultaneously using existing skew quadrupoles was developed. Measured coupling and dispersion functions acquired before and after correction are presented.

\section{THE MOTIVATION}

The Relativistic Heavy Ion Collider (RHIC) is the only hadron collider with a high energy polarized proton physics program in the world. It consists of two counter rotating accelerators (called Blue and Yellow). The figure of merit for physics experiment of polarized proton with longitudinal spin direction of both beam is $L \cdot P_{\text {Blue }}^{2} \cdot P_{\text {Yellow }}^{2}$ [1], where $L$ is the luminosity, and $P_{B l u e}$ and $P_{Y \text { ellow }}$ are the polarization of the Blue and Yellow beam respectively. During RHIC Run-11 $250 \mathrm{GeV}$ polarized proton collisions, measurements revealed a $10-15 \%$ loss of polarization during the ramp to full energy and a $10 \%$ loss over the course of an 6-hour store [2]. While efforts to improve presentation of the proton's polarization are ongoing, we considered here the possible correlation between polarization and vertical dispersion. Both vertical dispersion and depolarization may result from vertical deflections of the beam, which disturbs the spin axis to deviate from the vertical direction in a ideal machine. Therefore, correcting vertical dispersion might prove benificial in preserving beam polarization.

\section{KNOBS FOR VERTICAL DISPERSION CORRECTION}

Vertical dipole correctors and skew quadrupoles are two possible actuators that can be utilized to correct vertical dispersion. The choice between these two largely depends on how much dispersion can be generated by them compared with the residual dispersion in the machine. Offline analysis pointed to global skew quads as the dominant contributor for vertical dispersion, therefore, the proposed scheme employs skew quads as knobs to supress vertical dispersion.

${ }^{*}$ The work was performed under Contract No. DE-AC02-98CH10886 with the U.S. Department of Energy.

† cliu1@bnl.gov

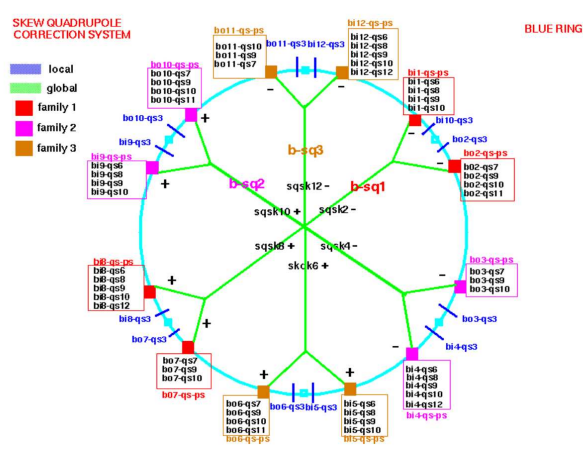

Figure 1: SQ families configuration in the blue ring

\section{SKEW QUADRUPOLE TOPOLOGY AT RHIC}

The configuration of skew quads in the Blue and Yellow ring is similar. In Fig. 1 the schematic of skew quads in Blue is shown [3]. Skew quads in the interaction regions are intended to correct local coupling arising primarily from roll errors of the triplet quadrupoles. There is one skew quad for each triplet (total 12). Skew quads (total 48) between the arc and interaction region are for correcting linear difference global coupling [4], which are grouped as three families according to their coupling contribution's direction. There are 12 power supplies for these 48 skew quads, of which those in any given colored box form a single family with common setpoints to their respective power supplies.

\section{DISPERSION RESPONSE TO SKEW QUADRUPOLES}

The self-consistent solution of dispersion when a skew quad strength changes by $\triangle(k l)$ is

$\triangle \eta_{y}=-\triangle(k l) \eta_{x} \cdot \frac{\sqrt{\beta_{y} \beta_{y 0}}}{2 \sin (\pi \cdot Q)} \cdot \cos \left(\pi \cdot Q-\left|\phi_{y 0}-\phi_{y}\right|\right)$

The response of dispersion to a unit change in skew quad strength can be expressed in matrix form as [5]

$$
\left(\begin{array}{c}
D_{1} \\
D_{2} \\
\vdots \\
D_{m}
\end{array}\right)=\left(\begin{array}{cccc}
R_{11} & R_{12} & \cdots & R_{1 n} \\
R_{21} & R_{22} & \cdots & R_{2 n} \\
\vdots & \vdots & \ddots & \vdots \\
R_{m 1} & R_{m 2} & \cdots & R_{m n}
\end{array}\right) *\left(\begin{array}{c}
k_{1} \\
k_{2} \\
\vdots \\
k_{n}
\end{array}\right)
$$

where $D_{i}$ is the dispersion at $i$ th BPM, $k_{j}$ is the integral strength of the $j$ th skew quad, and $R_{i j}=-\frac{\sqrt{\beta_{i} \beta_{j}}}{2 \sin (\pi \cdot Q)} \eta_{x}$. $\cos \left(\pi \cdot Q-\left|\phi_{i}-\phi_{j}\right|\right)$. 


\section{IMPACT ON COUPLING}

The linear difference coupling coefficient $C^{-}$[4] generated by skew quads is given by

$C^{-}=\frac{1}{2 \pi} \sum_{i} \sqrt{\beta_{i x} \beta_{i y}} k_{i} l_{i} \exp \left(i\left(\phi_{i x}-\phi_{i y}-2 \pi \triangle \cdot s_{i} / L\right)\right)$.

Here $\beta_{i x, i y}$ are the uncoupled betatron amplitude functions, $\phi_{i x, i y}$ are the uncoupled betatron phase advances, $k_{i}$ and $l_{i}$ are individual skew quadrupole strength and length, $L$ is the ring circumference, and $s_{i}$ is the distance between the skew quadruple and the reference point. Based on Eq. 3, a matrix describing the respone of linear difference coupling coefficient to skew quads strength for the 6-family case (skew quads around one IR form a family) is given by

$$
\left(\begin{array}{c}
\operatorname{Re}\left(C^{-}\right) \\
\operatorname{Im}\left(C^{-}\right)
\end{array}\right)=\left(\begin{array}{llllll}
N_{11} & N_{12} & N_{13} & N_{14} & N_{15} & N_{16} \\
N_{21} & N_{22} & N_{23} & N_{24} & N_{25} & N_{26}
\end{array}\right) *\left(\begin{array}{c}
k_{1} \\
k_{2} \\
k_{3} \\
k_{4} \\
k_{5} \\
k_{6}
\end{array}\right)
$$

\section{ALGORITHM OF SIMULTANEOUS COUPLING AND DISPERSION CORRECTION}

In order to correct coupling and dispersion simultaneously, one needs to solve linear equations for both coupling and dispersion. The tricky part is to scale (e.g. effectively weight) the two sets of equations properly. The matrix form for simultaneous coupling and dispersion correction for the 6-family case is

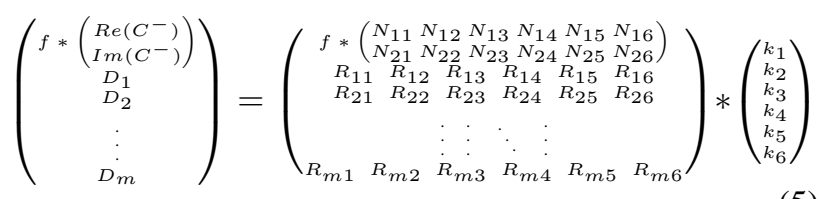

Here, $f$ is the scale factor, which weights the preference for coupling and dispersion correction.

\section{APPLICATION IN RHIC}

Algorithms for coupling correction and vertical dispersion correction were tested in RHIC separately before simultaneous correction. The weighting factor between two corrections are from offline test, which was again checked real time for online data.

\section{Coupling correction}

We first supress the coupling by applying routine coupling feedback which uses the existing 3-family scheme, then we calculate the coupling strength from the skew quads settings, then recalculate the required strength for the 12-family correction scheme. Coupling correction at injection and store have been applied for both rings successfully. Fig. 2 shows similar required strength for skew quads families before and after switching correction schemes, which is a sign that the new algorithm works as effective as the existing one. Fig. 3 shows the reduction of currents of skew quads.

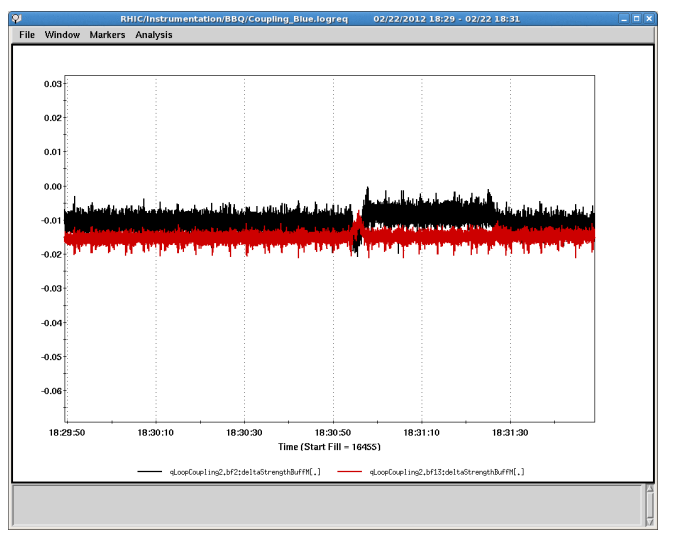

Figure 2: Required further strength for skew quads family (black for family 2, red for families 1 and 3) to reduce coupling to zero, which is also a representation of the coupling strength of the machine

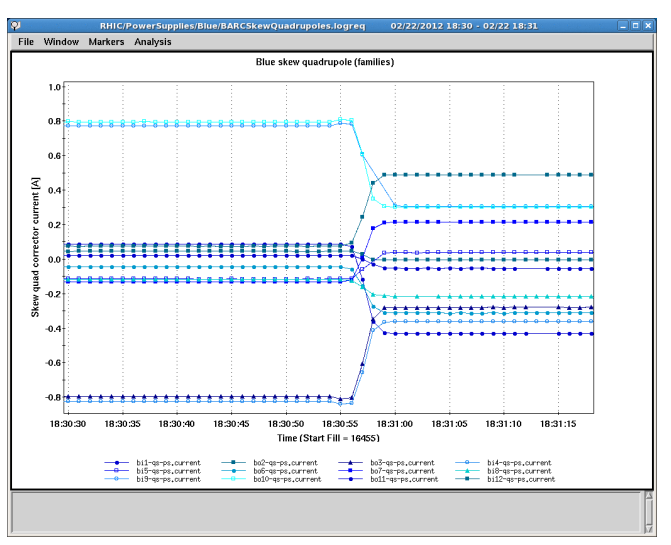

Figure 3: Skew quads currents before and after switching from a 3 -family to a 12-family coupling correction scheme

\section{Dispersion correction}

Baseline data for dispersion were acquired using the RhicChromaticity application. The dispersion response of BPMs to skew quads was measured to validate the calculation of the response matrix based on the online model. Due to larger discrepancies present in the IR region BPMs (BPMs within about $600 \mathrm{~m}$ around the IPs), the BPMs in IR regions are excluded from the calculation. About $50 \%$ reduction of vertical dispersion RMS (Fig. 4 and 5) were seen in both rings at injection. The corresponding change in coupling is shown in Fig. 6

\section{Simultaneous correction}

The scale factor $f$ in Eq. 5 for dispersion correction was set to be 0.002 as found to be optimum in both offline sim- 


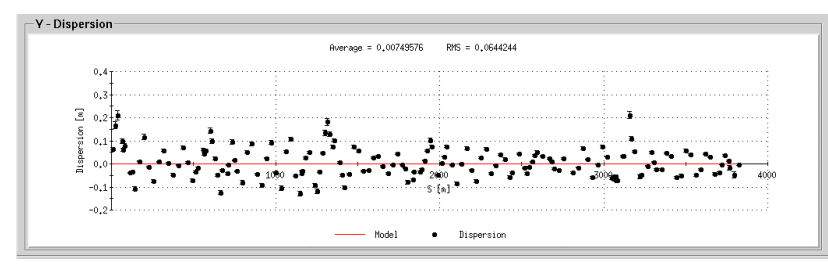

Figure 4: Baseline vertical dispersion data for correction in blue ring at injection energy, $\mathrm{rms}=0.064 \mathrm{~m}$

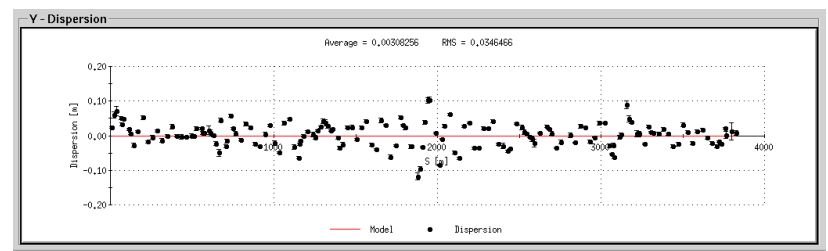

Figure 5: Vertical dispersion data after dispersion only correction in blue at injection, $\mathrm{rms}=0.035 \mathrm{~m}$

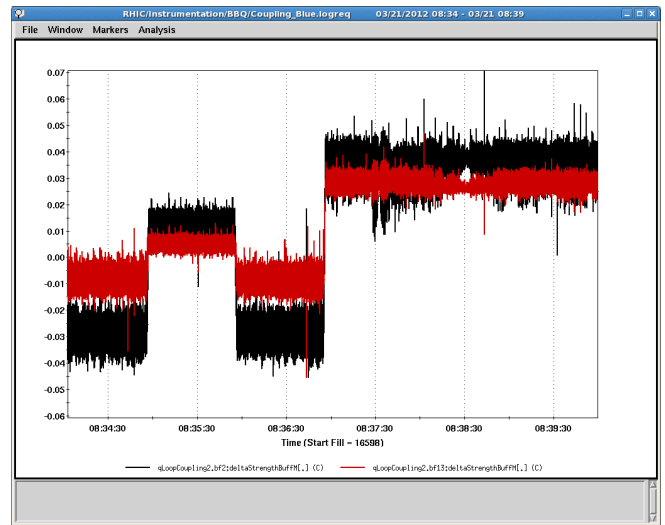

Figure 6: Coupling signals during the process of dispersion correction only, first increase of coupling was when $20 \%$ correction strength was put in, the second increase happened when we put in $50 \%$ correction

ulation and online experiment.

The coupling signals before and after correction as shown in Fig. 7 stayed more or less the same during simulaneous correction. The vertical dispersion before correction was shown in Fig. 4 and after simultaneous coupling and vertical dispersion correction is shown in Fig. 8, .

\section{CONCLUSION}

A new algorithm for simultaneously correcting coupling and vertical dispersion was proposed and successfully demonstrated in RHIC. The number of skew quadrupole families was increased from 2 to 12 . The skew quad strengths were redistributed to correct vertical dispersion while maintaining the global decoupling. A reduction of rms vertical dispersion by $\sim 50 \%$ was achieved in both

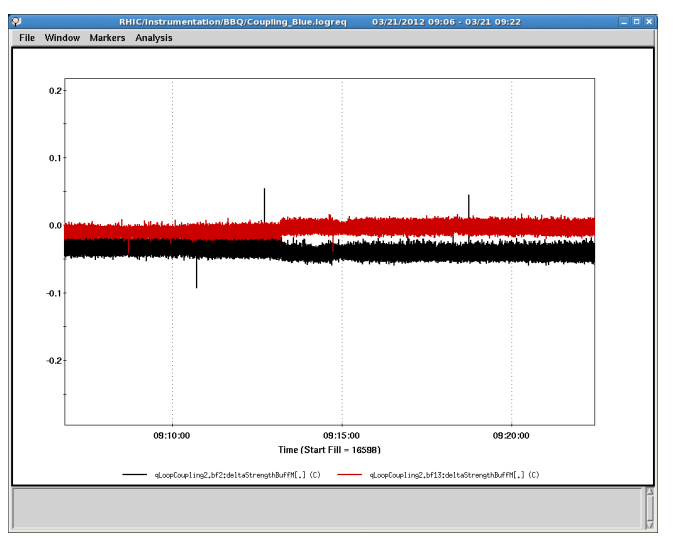

Figure 7: The coupling signal from skew quads family before and after implementation of simultaneous correction of coupling and dispersion

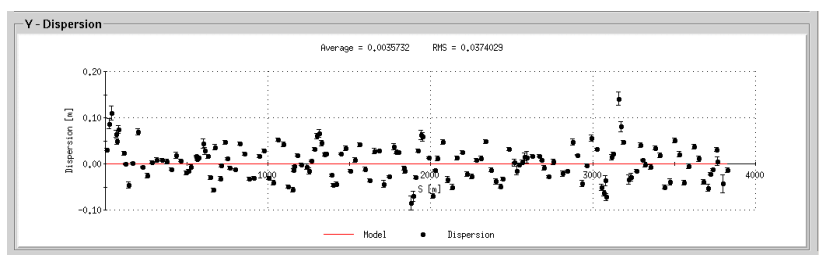

Figure 8: Vertical dispersion data after simultaneous coupling and dispersion correction in blue at injection, $\mathrm{rms}=$ $0.037 \mathrm{~m}$

rings. The remaining vertical dispersion will be corrected further by dispersion-free steering. Extension of this algorithm for the energy ramp will be explored in the future.

\section{REFERENCES}

[1] C. Montag, L. Ahrens, M. Bai, J. Beebe-Wang, M. Blaskiewicz, JM Brennan, KA Brown, D. Bruno, R. Connolly, T. DOttavio, et al. Rhic performance as a 100 gev polarized proton collider in run-9. Proceedings of IPAC10, pg, 531, 2010.

[2] H.Huang, L. Ahrens, I.G. Alekseev, E. Aschenauer, G. Atoian, et al. Rhic polarized proton operation. Proceedings of PAC11, 2011.

[3] F. Pilat, M. Bai, J. Beebe-Wang, J. Cardona, W. Fischer, and V. Ptitsyn. Coupling measurement and correction during rhic run 2001 and development for 2003. CA/AP, 77, 2002.

[4] Y. Luo, P. Cameron, A. Dellapenna, L. Hoff, A. Marusic, S. Peggs, C. Schultheiss, and R. Jones. Continuous measurement of global difference coupling using a phase-locked-loop tune meter in the relativistic heavy ion collider. Physical Review Special Topics-Accelerators and Beams, 9(12):124001, 2006.

[5] M. Aiba, R. Calaga, A. Franchi, R. Tomas, and G. Vanbavinckhove. Coupling and vertical dispersion correction in the sps. Proceedings of IPAC, 2010. 